\title{
Incidences socio-économiques et environnementales des infrastructures pastorales et agropastorales installées dans le Borgou au Nord-Est du Benin
}

\author{
I. M. ABDOULAYE ${ }^{1 *}$, M. AYENA ${ }^{2}$, A. J. YABI ${ }^{2}$, H. DEDEHOUANOU ${ }^{1}$, G. BIAOU ${ }^{3}$ et \\ M. HOUINATO ${ }^{4}$
}

\author{
${ }^{I}$ Département d'Economie, de Socio-anthropologie et de Communication pour le Développement Rural, \\ Faculté des Sciences Agronomiques, Université d'Abomey-Calavi ; 01 BP 526 Cotonou, Bénin. \\ ${ }^{2}$ Laboratoire d'Analyse et de Recherche sur les Dynamiques Economiques et Sociales (LARDES), Université \\ de Parakou;BP 23 Parakou, Bénin. \\ ${ }^{3}$ Université Nationale d'Agriculture de Porto-Novo, Bénin. \\ ${ }^{4}$ Faculté des Sciences Agronomiques de l'Université d'Abomey-Calavi; 01 BP 526 Cotonou, Bénin. \\ *Auteur correspondant ; E-mail : mamcherif@yahoo.fr
}

\section{RESUME}

Le développement du sous-secteur de l'élevage qui est d'une importance capitale dans la lutte contre la pauvreté a suscité la réalisation des ouvrages pastoraux et agropastoraux dans le Borgou, au Nord-Est du Benin en vue d'améliorer les conditions de vie des populations de cette zone. L'objectif du présent article est d'évaluer les effets socio-économiques et environnementaux de ces ouvrages sur les communautés les abritant. A cet effet, les données relatives aux volumes des facteurs de production des activités agricoles menées autour de ces ouvrages, aux volumes des outputs et à leurs prix de vente ; aux caractéristiques socio-démographiques des utilisateurs de ces ouvrages; aux conflits entre éleveurs et agriculteurs et au degré de pression sur les essences forestières ont été collectées à l'aide de questionnaires, de guides d'entretien et d'observations directes. Les statistiques descriptives, le test de Student, le test khi 2, la régression linéaire multiple et celle logistique binaire ont été utilisés pour analyser ces données. Les résultats montrent que les ouvrages pastoraux et agropastoraux impactent positivement les communautés. Les barrages et retenues d'eau aménagés, de par leur existence ont engendré le développement du maraîchage et de l'agriculture de contre saison. Les producteurs installés aux abords de ces infrastructures hydrauliques gagnent $32080 \mathrm{~F} \mathrm{CFA} / \mathrm{ha}$ de plus que leurs homologues installés ailleurs. Les éleveurs accédant à ces infrastructures gagnent 1003496 F CFA/an de plus que ceux n'y ayant pas accès. Les fréquences d'apparition de conflits entre éleveurs et agriculteurs ainsi que la pression sur les essences végétales à usage animal diminuent avec le traçage des couloirs de passage et la délimitation des aires de pâturage. Cependant, la durabilité des infrastructures hydrauliques et marchandes n'est pas garantie du fait de défaut de gestion. Les infrastructures de conduite pastorale ne sont non plus respectées tant par les agriculteurs que par les éleveurs. La mise en place d'un cadre réglementant l'usage de ces infrastructures serait d'une grande importance sociocommunautaire pour les localités les abritant.

(C) 2019 International Formulae Group. All rights reserved

Mots clés : Infrastructures pastorales et agropastorales, conflits, revenu, Borgou.

\section{Socioeconomic and environmental incidences of pastoral and agro-pastoral infrastructures implemented in Borgou, in North-East of Benin}

\section{ABSTRACT}

The development of the livestock sector, which plays an important role in the fighting against rural poverty, has led to the implementation of pastoral and agro-pastoral infrastructures in Borgou, in order to 
improve living conditions of the populations. Thus, this article aims to evaluate the socio-economic and environmental effects of these infrastructures on the communities where they were implemented. For this purpose, the data relating to the quantity of the factors of production of the agricultural activities carried out around hydraulic infrastructures, to the volumes of the outputs and their sales prices; to the socio-demographic characteristics of the users of these infrastructures; to the conflicts between pastoralists and farmers and the degree of pressure on forest species, were collected using questionnaires, interview guides and direct observations. Data were analyzed using descriptive statistics, student's t-test, chi-square test, multiple linear and binary logistic regressions. The results show that pastoral and agro-pastoral infrastructures have a positive impact on communities. The dams and reservoirs by their existence have led to the development of market gardening and off-season farming. Producers located near these hydraulic infrastructures earn 32,080 F CFA/ha more than their homologues installed elsewhere. Breeders accessing to these infrastructures earn $1003496 \mathrm{~F}$ CFA/year more than those without access. The frequency of conflict between farmers and breeders and the pressure on plant species for animal use decrease with the tracing of corridors and the delimitation of grazing areas. However, the sustainability of hydraulic and market infrastructure is not guaranteed due to management failure. The corridors and grazing areas's infrastructure are not respected either by the farmers as by the breeders. The establishment of a framework regulating the use of these infrastructures would be very important to socio-economic development of the localities where they were implemented.

(C) 2019 International Formulae Group. All rights reserved

Keywords: Infrastructures pastoral and agropastoral, conflict, revenue, Borgou.

\section{INTRODUCTION}

Au Benin, le sous-secteur de l'élevage est la deuxième activité économique après l'agriculture (Djenontin, 2010). Il contribue à hauteur de 6\% au PIB national (MAEP, 2010) et compte environ 1905000 têtes de bovins. Le cheptel national bovin est composé d'une diversité génétique constituée de races bovines Lagunaire, Somba, Borgou et des zébus, principalement, zébu Peul, White Fulani et M'Bororo Rouge (Youssao et al., 2013). La partie septentrionale du Benin est la zone d'élevage par excellence. On y rencontre diverses races (la race Somba dans l'Atacora, la race Borgou dans le Borgou et les zébus dans l'Alibori).

Le Département du Borgou de par ses conditions agro-écologiques favorables à l'élevage, a connu depuis plus d'une décennie, l'installation massive de plusieurs éleveurs venus du haut Nord, du Nigéria et du Niger. Ces éleveurs (sédentaires ou transhumants) pratiquent un mode d'élevage de type extensif et traditionnel basé sur l'exploitation du pâturage naturel (Youssao et al., 2013). Les expériences d'ici et d'ailleurs ont révélé que la coexistence des systèmes d'agriculture itinérante et d'élevage extensifs est source de divers problèmes d'ordre socio- environnemental. Pour Ibrahim (2016) ; Archambaud and Tidjani (2016); Ayantunde et al. (2014) et Umutoni et al. (2016), cette cohabitation crée une forte concurrence dans l'usage des ressources naturelles avec pour conséquence les conflits entre éleveurs et agriculteurs; la dégradation des essences végétales et la mise en culture des espaces réservés aux activités pastorales, réduisant ainsi les performances productives des deux secteurs d'activités. Le Département du Borgou n'échappe donc pas à cette réalité. De vastes superficies agricoles sont prises d'assaut par les éleveurs, de même que des espaces réservés au passage et au pâturage des animaux sont mis en culture, rendant donc difficile la cohabitation entre les éleveurs et les agriculteurs. Fort conscient de l'importance de ces secteurs dans le développement des collectivités locales qui devraient désormais se prendre en charge avec l'avènement de la décentralisation, diverses infrastructures pastorales et agropastorales y ont été installées et confiées aux Mairies. L'objectif de l'installation de ces infrastructures est de réduire les conflits entre agriculteurs et éleveurs d'une part et d'autre part, de permettre aux secteurs agricoles et de l'élevage d'amorcer leur développement. 
Alors, après plusieurs années d'utilisation de ces infrastructures, il s'est avéré crucial de procéder à leur évaluation en termes de gestion, d'effets/impacts engendrés, de durabilité afin de mettre dans un premier temps à la disposition des décideurs, des éléments nécessaires à la prise de bonnes décisions de réaménagement et dans un second, contribuer à l'animation des débats scientifiques sur les infrastructures pastorales et agropastorales.

Les travaux scientifiques ayant porté sur ces thématiques ont traité de l'état des lieux, de la caractérisation de ces infrastructures et de leurs modes de gouvernance (Tama et Mossi, 2011; Abdoulaye et al., 2018; Delville, 2006; Oyono et al., 2007 ; Sgahaier, 2010). Mais en termes d'évaluation d'effets socioéconomiques et environnementaux de ces infrastructures sur les collectivités bénéficiaires, peu d'études ont été réalisées. Le présent article est donc rédigé pour combler ce vide scientifique combien capital pour les prises de décisions de développement.

\section{MATERIEL ET METHODES}

\section{Cadre théorique de l'étude}

Le concept "incidence", encore désigné sous le vocable d'effets ou d'impacts (Brunet et al., 1993), désigne les répercussions, les conséquences plus ou moins directes d'une action, d'une intervention ou d'une politique sur un espace donné, sur une communauté donnée, sur un objet donné, etc. Ainsi, les incidences socio-économiques traduisent les conséquences, les effets, les répercutions plus ou moins directs d'une politique, sur la situation sociale et économique des populations d'un espace géographique donné.

L'effet/impact d'un projet ou d'un programme est défini comme l'ensemble des changements dans les conditions de vie des participants, tels qu'eux-mêmes et leurs partenaires les perçoivent au moment de l'évaluation, ainsi que tout changement durable dans leur environnement, auxquels le projet ou le programme a contribué. Ces changements peuvent être positifs ou négatifs, voulus ou imprévus (IFAD, 1998).

Plusieurs approches et méthodes, partant des postulats « sans-avec » ou « avantaprès », en fonction de la nature des sujets évalués, sont utilisées pour conduire les études d'impacts/d'effet. On y distingue l'approche naïve d'évaluation d'impact, l'approche expérimentale, quasi-expérimentale et celle non-expérimentale. Le choix de l'une de ces méthodes est déterminé par son coût d'application, sa faisabilité au regard des sujets évalués, le degré de clarté et de fiabilité des résultats auxquels elle conduit et le degré du biais de sélection.

L'approche naïve, la plus simple consiste à disposer de deux groupes distincts d'individus, dont l'un est le groupe des traités et le second, celui des témoins. L'estimation d'effet consiste donc à faire la différence entre les moyennes simples des résultats observés au niveau de chacun d'eux. Bien qu'étant fréquemment utilisée dans la littérature (Adekambi, 2005), cette approche est qualifiée de potentiellement biaisée (Heckman, 1990 ; Diagne, 2003) du fait qu'elle ne tient pas compte des caractéristiques des participants et d'autres facteurs qui influencent leur décision de participer ou non au programme.

L'approche expérimentale quant à elle suppose l'existence de deux groupes d'individus ayant les mêmes caractéristiques, assignés de façon aléatoire aux groupes et respectant scrupuleusement ces assignations respectives. La seule différence entre les deux groupes est le traitement c'est-à-dire la participation au programme (dans ce cas, l'usage ou non des infrastructures pastorales et agropastorales). L'assignation aléatoire des individus à un groupe ou à l'autre rend le traitement indépendant aux deux résultats potentiels. Autrement dit, l'utilisation des infrastructures pastorales et agropastorales ou la non utilisation est aléatoire et les variables $\mathrm{Y}_{1}$ et $\mathrm{Y}_{0}$ (qui ne sont rien d'autres que les outcomes des utilisateurs et non utilisateurs) 
sont indépendantes. Les biais de sélection se réduisent donc à zéro et l'estimateur est sans biais. Cette simplicité de l'estimation de l'effet sans biais avec la méthode expérimentale, est la raison pour laquelle elle est généralement considérée comme étant la plus fiable (estimation non biaisée) et donnant les résultats les plus faciles à interpréter (Cocharne and Rubin, 1973 ; Fall, 2006). Cependant, ce type d'évaluation est difficile à appliquer dans la pratique sur les êtres humains qui sont de nature incontrôlable et pose le problème d'éthique dans le cas des phénomènes sociaux (Diagne, 2003). Elle est également extensive et nécessite plus de temps surtout quand il s'agit de collecter les nouvelles données.

Sous-tendue par deux sous approches (la méthode de comparaison appariée et celle réflexive), l'approche quasi-expérimentale s'apparente à celle expérimentale. La première consiste à identifier le groupe des participants au programme et celui des non participants (groupe de comparaison) sur la base des mêmes caractéristiques socioéconomiques observables ou sur la base de celles susceptibles d'influencer les résultats du programme. Autrement dit, ces deux groupes doivent avoir les mêmes caractéristiques socio-économiques. Le groupe de comparaison peut être sélectionné avant l'intervention du programme (étude prospective) ou après l'intervention du programme (étude rétrospective). L'avantage de cette approche est qu'elle peut conduire à l'utilisation d'une base de données existante donc moins coûteuse. Les principales difficultés/insuffisances de l'approche sont relatives à la fiabilité des résultats. L'approche ne permettant pas de corriger définitivement le problème de biais de sélection, conduit donc aux résultats peu fiables. Aussi, cette approche est-elle statistiquement complexe et requiert une grande capacité/expertise en évaluation d'effet et en interprétation des résultats d'analyse.

La méthode d'évaluation d'effet la plus appropriée à cette approche est la méthode des scores de propension. Elle est utilisée lorsqu'il existe un nombre important de caractéristiques socio-économiques utilisables pour comparer le groupe des bénéficiaires et celui des non bénéficiaires. Le score de propension est la probabilité de participer au programme étant donné un vecteur $\mathrm{X}$ de variables observables. L'effet est déterminé par comparaison du résultat moyen des utilisateurs d'infrastructures pastorales à celui des non utilisateurs.

La méthode de comparaison réflexive utilise le contre-factuel qui est constitué sur la base de la situation des participants au programme avant son intervention. Ainsi, les résultats des bénéficiaires du programme sont comparés entre eux avant et après le programme. Cette méthode est utilisée lorsqu'il s'agit d'évaluer les programmes/projets qui couvrent toute une région ou une nation. Toute la population de la zone d'intervention du programme/projet y participe ; donc, il n'y a pas la possibilité de constituer le groupe de comparaison. La faiblesse de cette méthode est qu'elle attribue tout changement observé au niveau des participants au programme aux seuls effets du programme, alors qu'en réalité, plusieurs d'autres facteurs tels que l'environnement économique, social, climatique, etc. peuvent influencer le résultat. Autrement dit, cette méthode ne sépare pas l'effet du programme sur le résultat des participants de celui des autres facteurs externes. Cela pose alors le problème de fiabilité des résultats.

Généralement utilisée par les économistes en se basant sur les théories économiques et économétriques, l'approche non expérimentale ou contre-factuelle permet de minimiser les erreurs potentielles dans l'estimation d'effet (Diagne, 2003). L'approche non-expérimentale est utilisée lorsqu'il est difficile de constituer un groupe de contrôle. Ainsi, on peut comparer les participants au programme aux non participants en faisant appel à des méthodes statistiques pour contrôler les différences observées entre les deux groupes qui peuvent 
influencer l'indicateur d'effet indépendamment de la participation au programme. Il est aussi possible à l'aide d'une régression linéaire de contrôler l'influence d'autres facteurs socio-économiques (âge, sexe, expérience, etc.) sur l'indicateur d'effet (Diagne, 2003; Yabi et al., 2014). Cette approche d'évaluation est relativement moins coûteuse et facile d'usage, mais l'interprétation des résultats requiert de connaissances avérées en économétrie de la part du chercheur.

Dans la présente étude, les infrastructures pastorales et agropastorales (barrages et retenues d'eau aménagées ; marchés à bétails ; couloirs de passage et aires de pâturage) sont considérées comme des ouvrages dont l'usage engendrait des effets sur les différents utilisateurs. Au regard des caractéristiques des différentes méthodes d'évaluation d'impacts/effet énumérées cidessus et vu que les infrastructures concernées sont accessibles à toutes les populations des zones où elles sont installées, il a été utilisé dans cette étude, la méthode de comparaison réflexive combinée à la régression linéaire multiple en se basant sur le postulat "sansavec" pour estimer les effets de ces infrastructures sur les utilisateurs. Plusieurs indicateurs d'effet ont été utilisés dans cette étude en fonction de l'infrastructure concernée. L'effet socio-économique des marchés à bétails, des barrages et retenues d'eau aménagées a été évalué sur les bénéficiaires avec comme indicateur les revenus des agriculteurs et éleveurs. L'effet social et environnemental des couloirs de passage et aires de pâturage a été également évalué en se basant sur la fréquence d'apparition des conflits entre éleveurs et agriculteurs et le dégrée de pression sur les essences végétales utilisées dans l'alimentation des animaux.

\section{Zone d'étude et base de données}

Les communes de Nikki (plus précisément dans les villages de Nikki-Centre ; Biro et Sakabanssi), de N'Dali (Boko,
N'dali-Centre et Kori) et de Tchaourou (Alafiarou, Kaki-Koka et Boukoussera) ont servi de cadre d'étude pour le présent article. Ces communes ont été choisies pour le nombre élevé d'infrastructures pastorales et agropastorales et la forte concentration d'éleveurs de bovins.

L'unité de recherche regroupe les utilisateurs des différentes infrastructures pastorales et agropastorales objet de cette étude tels que les agriculteurs, les éleveurs, les autorités locales (mairies) et les sages/chefferies villageoises.

Les données relatives aux volumes des facteurs de production des activités agricoles et para-agricoles menées autour de ces ouvrages, aux volumes des outputs et à leurs prix de vente; aux caractéristiques sociodémographiques des utilisateurs de ces ouvrages; aux fréquences des conflits entre éleveurs et agriculteurs et au degré de pression sur les essences forestières, ont été collectées à l'aide de questionnaires structurés, de guides d'entretien et d'observations directes auprès de 292 personnes choisies à la fois de façon raisonnée (autorités locales et sages villageois) en fonction de leurs statuts et aléatoire (agriculteurs et éleveurs). La Figure 1 présente la zone d'étude.

\section{Analyses statistiques}

\section{Modélisation de l'effet des infrastructures pastorales et agropastorales}

Partant des postulats théoriques énumérés plus haut, l'impact/effet de ces différentes infrastructures a été mesuré en faisant la différence entre les moyennes des indicateurs des bénéficiaires. Le revenu a été utilisé comme indicateur de mesure de l'effet économique des barrages, retenues d'eau aménagées et marché à bétail sur les utilisateurs qui sont les agriculteurs et éleveurs. D'après Paraïso et al. (2012) ; Yabi et al. (2012) et de Dangbo et al. (2019), le revenu assimilé à la richesse créée par l'ensemble des spéculations produites par l'exploitant, peut se calculer comme 
suit : $M N_{i}=P B_{i}-\left(C V_{i}+C F_{i}\right)=M B_{i}-C F_{i}$ (en FCFA/ha) où

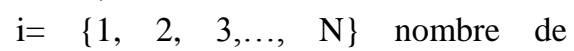
spéculations principales produites par le producteur. CV: coûts variables, correspondent aux dépenses liées à l'acquisition d'intrants (engrais, insecticides, pesticides et main d'œuvre). CF : coûts fixes, correspondent aux dépenses faites par l'exploitation mais non liées au volume de production; ce sont principalement les dépenses d'investissement. Les CF sont déterminés en appliquant un taux d'amortissement linéaire à la valeur totale d'acquisition du matériel. Ce taux correspond à l'inverse de la durée de vie de ce matériel.

En s'inspirant des travaux de Savi et al. (2008) et de Sounon et al. (2019), le revenu des éleveurs a été calculé ainsi qu'il suit: Rev $=$ PV - PR - CF (F CFA/tête).

PV : prix de vente d'un animal ; PR : prix de revient de l'animal ; CF : correspond aux charges fixes; elles englobent les taxes payées par les éleveurs, les redevances payées suite à l'utilisation des infrastructures pastorales et agropastorales, etc. Le prix de revient quant à lui est déterminé comme suit : $\mathrm{PR}=\mathrm{PA}+\mathrm{CT}$

PA : prix d'achat de l'animal. Il est égal à 0 pour l'animal ayant été mis bas au sein du troupeau ; CT : correspond au coût de traitement de l'animal. Ce coût englobe les dépenses liées aux soins de santé apportés à l'animal (vaccination, et autres dépenses de soins), aux dépenses relatives aux additifs alimentaires (apport d'éléments minéraux) depuis sa naissance ou son adhésion au troupeau jusqu'au jour de la vente. Ont été pris en compte dans le calcul du revenu, les animaux âgés d'au moins trois (03) ans.

Après estimation de ces revenus, nous avons calculé la différence entre la moyenne des revenus des agriculteurs et éleveurs avant et après l'accès aux infrastructures pastorales et agropastorales pour apprécier l'effet de l'utilisation de ces infrastructures sur eux. Le test $\mathrm{t}$ de Student a été effectué pour tester la significativité de la différence. Cette différence n'étant pas exclusivement due aux seuls effets des infrastructures, nous avons fait une régression linéaire multiple incluant d'autres facteurs (environnementaux, sociodémographiques des acteurs) pour extraire l'influence de chacun d'entre eux sur celle-ci. Sur la base des travaux de (Yabi et al., 2014), le modèle utilisé se présente sous la forme suivante.

$$
I_{m}=\sum_{i=1}^{k} \beta_{i} X_{i m}
$$

Im : indicateur de mesure de l'effet (revenu des agriculteurs et éleveurs) ;

$\beta_{\mathrm{i}}$ : coefficients des variables introduites dans le modèle et renseignant sur l'influence de chacune d'elles sur la variable dépendante ;

$\mathrm{X}_{\mathrm{im}}$ : vecteurs de variables explicatives composés de caractéristiques sociodémographiques des enquêtés, des facteurs environnementaux et d'une variable binaire $D$, renseignant sur la période d'évaluation. Elle prendra la valeur 0 pour la période avant utilisation des infrastructures et 1 pour la période après leur utilisation.

Mathématiquement, le modèle peut s'écrire comme suit :

\section{$\checkmark$ Au niveau des agriculteurs}

$R N_{\mathrm{i}}=\delta_{0}+\delta_{1} \mathrm{AGE}_{\mathrm{i}}+\delta_{2} S E X E_{\mathrm{i}}+\delta_{3} \mathrm{EDU}_{\mathrm{i}}+$ $\delta_{4}$ INFRA $_{\mathrm{i}}+\delta_{5} \mathrm{EXP}_{\mathrm{i}}+\delta_{6} \mathrm{CVULGA}_{\mathrm{i}}+$ $\delta_{7} \mathrm{ACRED}_{\mathrm{i}}+\mathrm{e}_{\mathrm{i}}$

avec

$R N$ revenu des agriculteurs; $\mathrm{e}_{\mathrm{i}}$ terme d'erreur aléatoire suivant une distribution normale avec une moyenne nulle et un écart-type constant. $\delta_{\mathrm{i}}$ les paramètres à estimer qui renseignent sur l'effet de chacune des variables sur le revenu.

AGE : est l'âge du producteur exprimé en nombre d'année; SEXE variable binaire correspondant au sexe de l'enquêté. Elle prend 1 s'il s'agit d'un homme et 0 si c'est une femme; EDU, renseigne sur l'éducation de l'enquêté, elle prend la valeur $=0$ si l'enquêté n'a pas reçu une éducation formelle et 1 s'il a été instruit. INFRA : variable binaire renseignant sur l'accès ou non aux infrastructures. Elle prend la valeur 1 si 
l'enquêté accède aux infrastructures et 0 si non. EXP correspond au nombre d'années d'expérience de l'enquêté dans l'activité, exprimée en nombre d'année. CVULGA: représente le contact avec un agent de vulgarisation. CONTAC $=0$ si l'enquêté n'est pas en contact avec un agent vulgarisateur, et CONTAC $=1$ s'il est en contact avec un agent vulgarisateur. ACRED : accès au crédit, $\mathrm{ACRED}=0$ si non et $\mathrm{ACRED}=1$ si oui.

\section{$\checkmark$ Au niveau des éleveurs}

Le modèle se présente comme suit :

$R N_{\mathrm{i}}=\delta_{0}+\delta_{1} \mathrm{AGE}_{\mathrm{i}}+\delta_{2}$ EPIZ $_{\mathrm{i}}+$ $\delta_{3} P{ }^{2} U V I O_{\mathrm{i}}+\delta_{4} B A R A G+\delta_{5} \mathrm{AMAR}_{\mathrm{i}}+$ $\delta_{6} \mathrm{AVET}_{\mathrm{i}}+\delta_{7} C A P A_{\mathrm{i}}+\mathrm{e}_{\mathrm{i}}$

avec

$R N_{\mathrm{i}}$ revenu de l'éleveur $\mathrm{i} ; \delta$ et e sont identiques à ceux décrits plus haut.

AGE : variable continue, correspondant à l'âge de l'éleveur, exprimé en nombre d'année. EPIZ: variable binaire renseignant sur l'apparition des épizooties au sein du troupeau. Elle prend la valeur 1 s'il y a eu attaque des animaux et 0 si non.

PLUVIO : cette variable renseigne sur l'état du climat (bon ou mauvais), mesuré par la pluviométrie. Elle prend la valeur 1 s'il y a une bonne pluviométrie (moyenne de 1000 $\mathrm{mm} / \mathrm{an}$ et plus pour la période) et 0 si la pluviométrie est mauvaise (en dessous de $1000 \mathrm{~mm} / \mathrm{an}$ ). La pluie a un impact direct sur la disponibilité en fourrage qui à son tour influence la performance des animaux. BARAG: variable binaire renseignant sur l'utilisation des infrastructures (barrage). Elle prend la valeur 0 pour les non utilisateurs et 1 pour les utilisateurs. AMAR : variable binaire renseignant sur l'accès au marché. Elle prend la valeur 1 si l'éleveur accède facilement au marché et 0 si non. AVET : variable binaire, traduisant l'accès aux produits vétérinaires. Elle prend la valeur 1 si l'éleveur accède et utilise les produits vétérinaires et 0 si non. CAPA: variable dichotomique renseignant sur le contact avec un technicien en production animale. CAPA prend la valeur 1 si l'éleveur a accès à un technicien en production animale et 0 si non.
Les variables pour lesquelles $\delta_{\mathrm{i}}$ est positif et significatif au seuil de $5 \%$ ont un effet positif sur les revenus des acteurs concernés.

La fréquence d'apparition de conflits et l'état physique des peuplements des essences forestières à usage animal ont été utilisés pour évaluer respectivement les effets social et environnemental des couloirs de passage et des aires de pâturage sur les bénéficiaires. L'état physique a été apprécié par les producteurs en termes de peuplement «pas du tout dégradé » où les essences se développent normalement sans aucune menace; peuplement «moyennement dégradé » où les essences subissent déjà les incidences négatives des actions anthropiques et peuplement «fortement dégradé » où les essences sont fortement menacées et sont en voie de disparition.

Ces indicateurs ont été déterminés aussi bien pour les zones abritant ces ouvrages que pour celles ne les abritant pas. Les fréquences de ces indicateurs ont été comparées entre elles selon l'existence ou non de couloirs de passage et d'aire de pâturage. Ensuite, une régression logistique binaire a été réalisée pour identifier les autres facteurs susceptibles d'influencer l'apparition ou non de conflits entre éleveurs et agriculteurs dans une aire géographique donnée. Le modèle logistique utilisé se présente comme suit :

$$
\begin{aligned}
\text { CONF }_{\mathrm{i}}=\beta_{0}+\beta_{1} \text { COMITRANS }_{\mathrm{i}}+\beta_{2} \text { EMORGEL }_{\mathrm{i}} \\
+\beta_{3} \text { LOISCHA }_{\mathrm{i}}+\beta_{4} \text { AIREPAT }_{i} \\
+\beta_{5} \text { CPASS }_{\mathrm{i}}+\mathrm{e}_{\mathrm{i}}
\end{aligned}
$$

CONF: variable qualitative binaire renseignant sur l'apparition ou non de conflits. Elle prend la valeur 1 s'il y a eu au moins un conflit entre agriculteurs et éleveurs au cours d'une année et 0 si non. Elle est la variable dépendante. COMITRANS : variable binaire dichotomique renseignant sur l'existence ou non de comité de transhumance. Cette variable prend la valeur 1 si oui et 0 si non. EMPRGEL, également qualitative et binaire, cette variable représente l'émergence d'organisations professionnelles au sein des éleveurs. Elle prend la valeur 1 si oui et 0 si non. LOISCHA, variable qualitative 
dichotomique renseignant sur l'existence des lois encadrant la cohabitation entre éleveurs et agriculteurs. Elle prend la valeur 1 si oui et 0 si non. AIREPAT, variable renseignant sur l'existence d'une aire de pâturage. AIREPAT prend la valeur 1 s'il y en a et 0 si non. CPASS : renseigne sur l'existence des couloirs de passage. Elle prend la valeur $1 \mathrm{~s}$ 'il y a dans la zone de couloirs de passage et 0 si non. $e_{i}$ est le terme d'erreur aléatoire suivant une distribution normale avec une moyenne nulle et un écart-type constant. $\beta$ correspond aux paramètres à estimer qui renseignent sur l'effet de chacune des variables sur celle dépendante.
La corrélation de rang utilisant le rho (p) de Spearman a été utilisée pour analyser l'évolution de l'état physique du peuplement avec la délimitation des aires de pâturage et des couloirs de passage. L'état physique des peuplements des essences forestières a été apprécié à travers les perceptions des enquêtés sur le niveau de dégradation des essences considérées, regroupées en trois catégories telles «peuplement pas du tout dégradé »; «peuplement moyennement dégradé» et «peuplement fortement dégradé ». Toutes ces analyses ont été faites avec le logiciel SPSS 20.

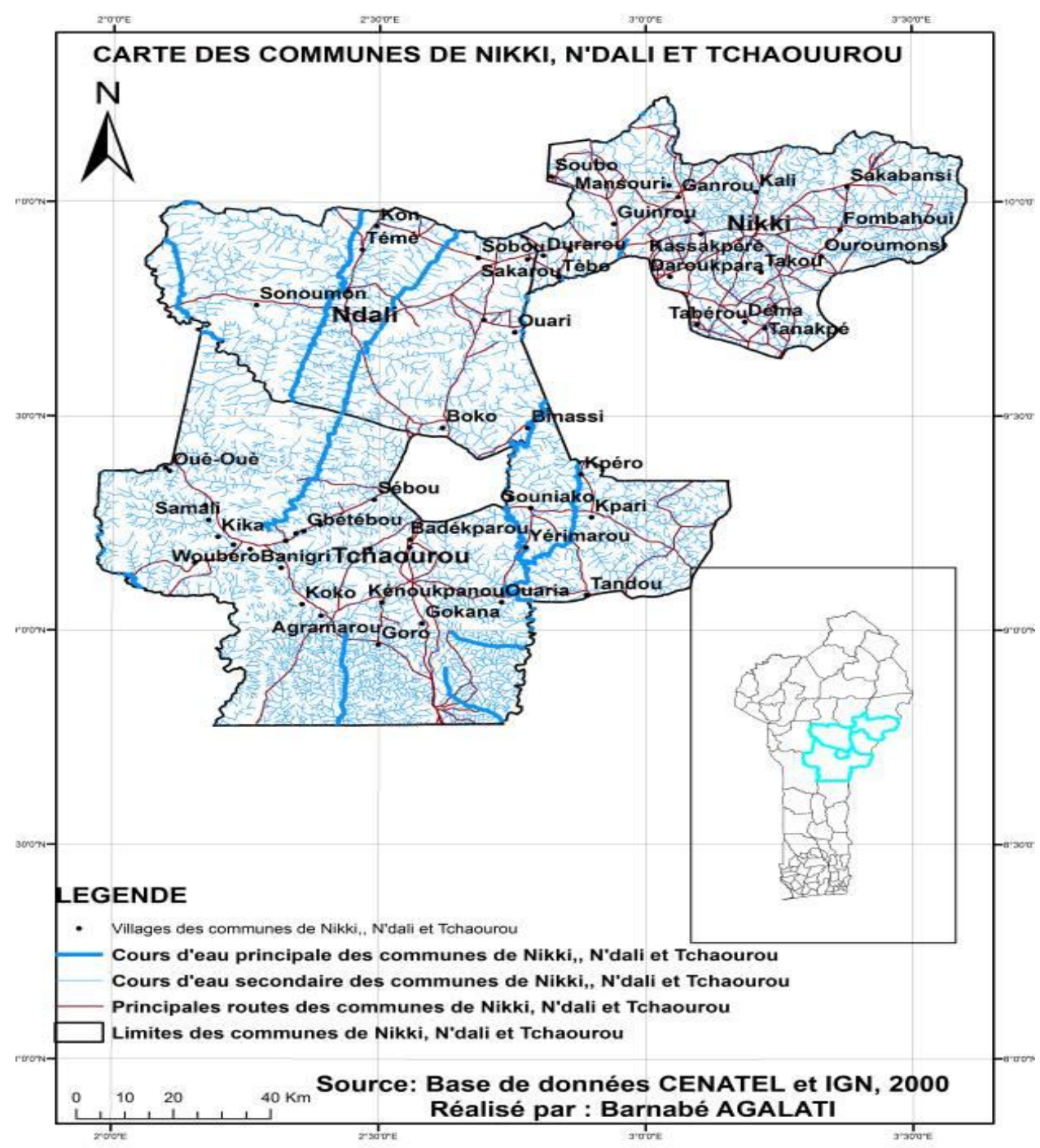

Figure 1 : Carte de la zone d'étude. 


\section{RESULTATS}

\section{Statistiques descriptives des variables introduites dans les modèles}

D'une moyenne d'âge de 42 ans (avec un écart-type de 10 ans), les producteurs (trices) enquêtés, majoritairement du sexe masculin (84,9\% des cas), témoignent d'une expérience moyenne de 16 ans dans la production agricole. Avec un taux d'accès à l'infrastructure (barrage et retenues d'eau aménagées) de 78,5\%, les producteurs (trices) dégagent un bénéfice moyen de $88750 \mathrm{~F}$ CFA par ha (43 640 F CFA d'écart-type) de leurs activités agricoles (maraîchage) menées autour de ces infrastructures malgré leur faible taux d'accès aux crédits $(8,1 \%$ des cas). Quarante-huit pourcent $(48,4 \%)$ d'entre eux, bénéficient des appuis des agents de vulgarisation.

Les éleveurs quant à eux, moyennement âgés de 46 ans (10 ans écarttype), font un bénéfice annuel moyen de trois millions vingt-quatre mille cent quatre-vingtdix-huit (3 024 198) F CFA principalement issu de la vente des produits et sous-produits du bétail. Cinquante-cinq pourcent $(55,3 \%)$ d'entre eux accèdent aux infrastructures hydrauliques (barrages et retenues d'eau aménagées) contre $43,5 \%$ qui accèdent aux marchés à bétails. L'apparition d'épizooties a été notifiée chez $31,8 \%$ des cas pendant que $72,9 \%$ déclarent avoir accès aux produits vétérinaires pour les divers soins même s'ils ne sont pas tous certifiés. Quarante-sept pourcent $(47,1 \%)$ des producteurs de l'échantillon qualifient l'état du climat de bon contre $52,9 \%$ qui pensent le contraire. La majorité des éleveurs enquêtés reçoivent l'appui de technicien en production animale. Les Tableaux 1 et 2 ci-dessous récapitulent les statistiques descriptives des variables introduites dans les modèles de régression.

\section{Effets socio-économiques des infrastructures hydrauliques et marchandes sur les utilisateurs}

L'analyse comparée des revenus des producteurs ayant accès aux barrages et retenues d'eau aménagées à celui de ceux n'y ayant pas accès, met en évidence une différence de valeur significative au seuil de $1 \% \quad(\mathrm{p}=0,0001 ; \mathrm{t}=4,65 ; \mathrm{ddl}=69,47)$. Les producteurs accédant aux barrages et aux retenues d'eau gagnent $32080 \mathrm{~F} \mathrm{CFA/ha} \mathrm{de}$ plus que leurs homologues n'ayant pas accès à ces infrastructures.

Tout comme au niveau des producteurs, les éleveurs accédant aux infrastructures hydrauliques ont des bénéfices plus importants ( $3472730 \mathrm{~F} \mathrm{CFA}$ ) que leurs homologues n'ayant pas accès (2 $469234 \mathrm{~F}$ CFA). La différence entre les deux groupes qui s'établit à 1003496 F CFA est significative au seuil de $10 \% \quad(\mathrm{p}=0,09$; $\mathrm{t}=1,693 ; \mathrm{ddl}=83$ ). Les Figures 2 et 3 illustrent les revenus par statut et par types d'acteurs.

De l'analyse de ces graphes, on en déduit que les infrastructures hydrauliques (barrages et retenues d'eau aménagées) ont un impact positif tant sur les producteurs que sur les éleveurs. Cet impact positif traduit par l'augmentation des revenus de ces derniers, ne saurait être entièrement expliqué par les seuls effets desdites infrastructures. Il existerait d'autres facteurs socioéconomiques susceptibles d'influencer au même titre que les infrastructures, les revenus des producteurs et éleveurs.

Pour ce qui est des producteurs, les résultats indiquent que le modèle de régression linéaire multiple réalisé à cet effet est globalement significatif au seuil de $1 \%$. Quarante-trois pourcent $(43,2 \%)$ des variations des revenus des producteurs sont expliquées par les variations des variables indépendantes introduites dans le modèle. Les variables «accès aux barrages et retenues d'eau aménagées », « accès à l'éducation formelle », «accès aux crédits » sont positivement significatives au seuil de $1 \%$ tandis que la variable « contact avec un agent de vulgarisation » est positive et significative au seuil de $10 \%$ (Tableau 3).

L'accès aux barrages et retenues d'eau a donc un effet positif sur le revenu des producteurs. Lorsqu'on passe d'un producteur maraîcher installé auprès d'un barrage ou d'une retenue d'eau aménagée à celui installé ailleurs, le revenu du producteur 
augmente de 32123 F CFA par hectare. Avoir accès à un agent de vulgarisation fait augmenter le revenu du producteur de 11611 F CFA comparativement à celui n'ayant aucun contact avec un encadreur. L'éducation formelle agit aussi positivement sur le revenu des agriculteurs. En passant d'un producteur non instruit à celui qui l'est, le revenu augmente de $15600 \mathrm{~F}$ CFA. L'accès au crédit influence positivement les revenus des agriculteurs. Il entraîne une augmentation à hauteur de 31113 F CFA lorsqu'on passe d'un producteur n'ayant pas accès au crédit à celui qui en a accès.

D'un pouvoir explicatif de $39,1 \%$ et significatif au seuil de $1 \%$, le modèle linéaire multiple utilisé aux fins d'identifier d'autres facteurs influençant les revenus des éleveurs montrent que l'âge de l'éleveur, le contact avec un technicien en production animale, l'accès aux barrages et retenues d'eau aménagées, l'état du climat sont les variables qui agissent positivement sur leurs revenus (Tableau 4).

L'âge du producteur, positivement significatif au seuil de 5\%, fait augmenter le revenu de $63398 \mathrm{~F}$ CFA lorsqu'on passe d'un jeune éleveur à l'éleveur plus âgé. Egalement positif et significatif au seuil de $5 \%$, la variable contact avec un technicien en production animale fait augmenter le revenu de 1210619 F CFA. L'accès aux barrages et retenues d'eau aménagées engendre une augmentation des revenus des éleveurs de 957456 F CFA lorsqu'on passe d'un éleveur qui n'a pas accès à ces infrastructures à celui qui en a accès. L'état du climat (mesuré ici à travers la pluviométrie) influence positivement le revenu des éleveurs. Lorsqu'on passe d'une mauvaise saison (manque de pluie) à celle bonne, le revenu des éleveurs augmente de 1131878 F CFA. Les Tableaux 3 et 4 présentent les résultats des différents modèles de régression linéaire.

\section{Effets sociaux et environnementaux des couloirs de passage et aires de pâturage \\ $\checkmark$ Effets sociaux}

Les infrastructures pastorales et agropastorales ont des effets sociaux positifs sur les communautés les abritant. Les fréquences d'apparition des conflits entre éleveurs et agriculteurs ont diminué avec le traçage des couloirs de passage et la délimitation des aires de pâturage. L'existence de ces infrastructures réduit considérablement les fréquences d'envahissement des champs par les troupeaux. Lequel envahissement créait fréquemment les discordes entre les éleveurs et agriculteurs.

Toutefois, la raréfaction du fourrage dans les aires de pâturage provoque le débordement des animaux des zones réservées à cette fin. Ce qui expliquerait quelques tensions entre éleveurs et agriculteurs qui continuent de subsister de nos jours. Les Figures 4 et 5 présentent les fréquences d'apparition de conflits selon l'existence ou non d'infrastructures pastorales et agropastorales.

De l'analyse de ces graphes, il ressort que dans les localités sans aires de pâturage, $40 \%$ des éleveurs déclarent avoir eu des conflits avec les agriculteurs au moins une fois au cours d'une année tandis que 15\% seulement l'ont eu dans les zones où elles ont été délimitées. De même, 45,2\% des éleveurs ont eu au moins un conflit avec les agriculteurs au cours d'une année dans les zones où il n'y a pas de couloirs de passage contre 11,6\% d'éleveurs dans les localités à couloirs de passage. Il pourrait donc en découler que ces infrastructures pastorales et agropastorales réduisent le taux d'apparition des conflits entre éleveurs et agriculteurs et contribuent de ce fait à l'apaisement du climat social entre les agriculteurs et éleveurs d'une même aire géographique donnée.

Ces deux infrastructures n'étant pas suffisantes à elles seules pour expliquer cette diminution de conflits entre éleveurs et agriculteurs, un modèle logistique binaire a été estimé afin de mettre en exergue d'autres facteurs susceptibles d'influencer cette baisse du taux d'apparition des conflits. Significatif à $1 \%$ avec un pouvoir explicatif de $39,6 \%$, le modèle logistique binaire révèle que l'existence de comité de transhumance, l'existence d'aires de pâturage et de couloirs 
de passage réduisent la probabilité d'apparition des conflits entre éleveurs et agriculteurs. La variable existence de comité de transhumance, négativement significative au seuil de $5 \%$, réduit de $61,9 \%$ la probabilité d'apparition de conflits entre éleveurs et agriculteurs. Comme révélé plus haut, le modèle vient confirmer les corrélations négatives existantes entre l'apparition de conflits et les infrastructures pastorales. Toutes deux (aires de pâturage et couloirs de passage) réduisent respectivement la fréquence d'apparition de conflits entre éleveurs et agriculteurs de $61,9 \%$ et $65,5 \%$ lorsqu'on passe d'une zone sans ces infrastructures à celle où elles sont installées. Le Tableau 5 récapitule les résultats du modèle de régression logistique binaire.

\section{$\checkmark \quad$ Effets environnementaux}

En ce qui concerne l'incidence environnementale des infrastructures pastorales et agropastorales, il convient de noter qu'il a été mis en évidence une corrélation positive entre l'existence des aires de pâturage et couloirs de passage et l'état physique des peuplements forestiers à usage animal. L'état physique des essences forestières à usage animal s'améliore avec l'installation de ces infrastructures. Plus d'acteurs (soit $44,4 \%$ pour les aires de pâturage et $33,3 \%$ pour les couloirs de passage) perçoivent les peuplements d'essences forestières à usage animal comme étant plus dégradés dans les espaces où il n'y a pas de telles infrastructures que dans les localités les abritant. La délimitation des aires de pâturage et de couloirs de passage réduit le nombre d'essences exposées aux bouviers qui pratiquent fortement le pâturage aérien surtout en saison sèche. Les seules pressions exercées sur les essences sont celles issues des actions humaines (installation de nouveaux champs, utilisation médicinales des plantes, etc.) qui ont commencé par diminuer de nos jours suite à la prise de conscience grandissante sur la préservation des ressources naturelles. Les Figures 6 et 7 illustrent la perception des populations sur l'état physique des peuplements des essences forestières à usage animal en fonction de l'existence ou non des infrastructures. De l'analyse de ces graphes, il est constaté que lorsqu'on passe d'une zone ayant délimité l'aire de pâturage à celle ne l'ayant pas fait, le taux de peuplement pas du tout dégradé passe de $15,6 \%$ à $52,5 \%$. Pour ce qui concerne les couloirs de passage, ce même taux passe de $31 \%$ à $34,9 \%$. Ainsi, on en déduit que les couloirs de passage et aires de pâturage contribuent à la préservation des essences forestières à usage animal et par ricochet à la protection de l'environnement. Sauf que les couloirs de passage le font moins bien que les aires de pâturage. La corrélation de rang recherchée entre l'état physique des peuplements forestiers à usage animal et autres facteurs susceptibles de l'expliquer tels que les feux de brousse, les actions anthropiques révèlent tout de même une corrélation négative. Ceci dit, les feux de brousse et les actions humaines (agriculture, usage aux fins sanitaires, domestiques, etc.) contribuent également à la dégradation de ces essences.

\section{Autres effets des infrastructures pastorales et agropastorales}

Les infrastructures pastorales et agropastorales notamment les marchés à bétails et barrages en plus de leurs effets positifs sur les revenus des agriculteurs et éleveurs, contribuent également au développement communautaire des localités les abritant. De par les recettes qu'elles génèrent aux mairies, elles constituent une source non négligeable dans l'alimentation de leurs budgets. Elles contribuent de ce fait directement ou indirectement à la réalisation des ouvrages sociocommunautaires (écoles, hôpitaux, marché, pistes rurales, etc.) qui sont d'une importance cruciale dans le développement économique et social de ces localités. Aussi, ces infrastructures notamment les barrages et retenues d'eau contribuent-elles à l'amélioration de la qualité nutritionnelle des aliments des populations environnantes à travers les produits halieutiques qu'elles offrent. 
Tableau 1 : Statistiques descriptives des variables introduites dans le modèle des producteurs.

\begin{tabular}{lcc}
\hline \multicolumn{3}{c}{ Variables quantitatives } \\
\hline Revenu net producteurs (F CFA/ha) & \multicolumn{2}{c}{$88750( \pm 43640)$} \\
Age (année) & $42( \pm 10)$ \\
Expérience & $16( \pm 8)$ \\
& Variables qualitatives (en \%) & \\
Infrastructure & Oui & 78,5 \\
& Non & 21,5 \\
Sexe & M & 84,9 \\
& F & 15,1 \\
Obtention du crédit & Oui & 8,1 \\
& Non & 91,9 \\
Education formelle & Oui & 38,7 \\
Contact avec un agent vulgarisateur & Non & 61,3 \\
& Oui & 48,4 \\
& Non & 51,6 \\
\hline
\end{tabular}

Tableau 2 : Statistiques descriptives des variables introduites dans le modèle des éleveurs.

\begin{tabular}{lcc}
\hline \multicolumn{3}{c}{ Variables quantitatives } \\
\hline Revenu annuel net éleveurs (F CFA) & $3024108( \pm 2746647)$ \\
Age (année) & Variables qualitatives (en \%) & \\
& Oui & \\
& Non & 55,3 \\
Accès barrage & Oui & 44,7 \\
& Non & 43,5 \\
Accès au marché à bétail & Oui & 56,5 \\
& Non & 31,8 \\
Apparition épizootie & Bon & 68,2 \\
& Mauvais & 47,1 \\
Etat du climat & Oui & 52,9 \\
& Non & 58,8 \\
Contact avec un technicien en production animale & Oui & 41,2 \\
Accès aux produits vétérinaires & Non & 72,9 \\
\end{tabular}




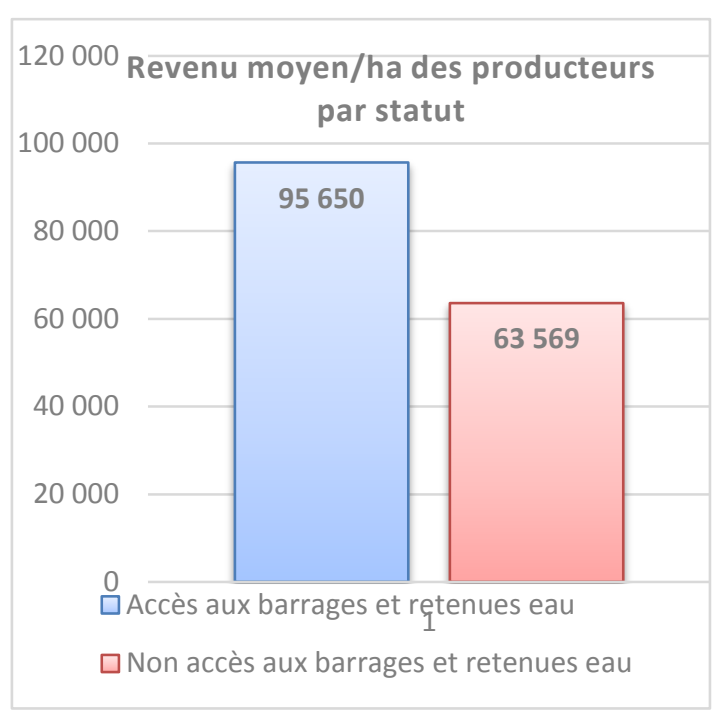

Figure 2 : Distribution des revenus des producteurs selon l'accès ou non aux infrastructures hydrauliques.

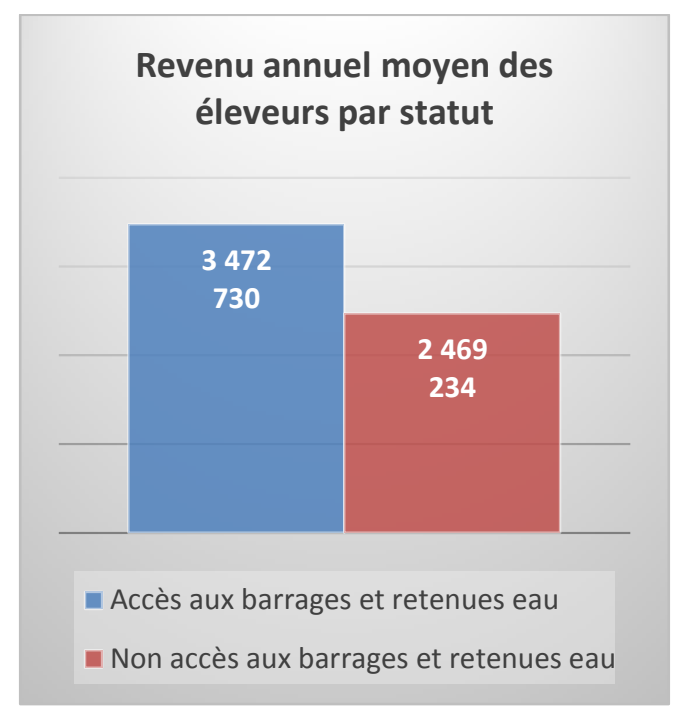

Figure 3 : Distribution des revenus des éleveurs selon l'accès ou non aux infrastructures hydrauliques.

Tableau 3 : Résultats du modèle des producteurs.

\begin{tabular}{|c|c|c|c|c|}
\hline Variables & Coefficients & Erreur standard & Statistique $\mathbf{t}$ & Signification \\
\hline Variable dépendante & \multicolumn{4}{|c|}{ Revenu des producteurs (F CFA/ha) } \\
\hline Constante & 46433,379 & 18030,632 & 2,575 & 0,011 \\
\hline $\begin{array}{l}\text { Contact avec un agent de } \\
\text { projet }\end{array}$ & $11611,616 *$ & 5985,763 & 1,940 & 0,054 \\
\hline Accès ou non au crédit & $31113,158 * *$ & 11091,763 & 2,805 & 0,006 \\
\hline Accès au barrage & $32123,454 * *$ & 7390,101 & 4,347 & 0,000 \\
\hline Education formelle & $15600,155^{*}$ & 6420,787 & 2,430 & 0,016 \\
\hline $\begin{array}{l}\text { Nombre d'année } \\
\text { d'expérience }\end{array}$ & 426,257 & 539,818 & ,790 & 0,431 \\
\hline Age & $-373,446$ & 419,776 &,- 890 & 0,375 \\
\hline Sexe & 10473,546 & 9753,065 & 1,074 & 0,284 \\
\hline Récapitulatif du modèle & \multicolumn{4}{|c|}{$\begin{array}{c}\mathbf{R}^{2}=0,432 \\
\mathbf{P}=0,00001 * * \\
\mathbf{N}=186\end{array}$} \\
\hline
\end{tabular}

Tableau 4 : Résultats du modèle des éleveurs.

\begin{tabular}{lcccc}
\hline Variables & Coefficients & Erreur standard & Statistique t & Signification \\
\hline Variable dépendante & & \multicolumn{2}{c}{ Revenu des éleveurs (F CFA) } & \\
\hline Constante & $-1635824,942$ & 1537502,583 & $-1,064$ & 0,291 \\
$\begin{array}{l}\text { Contact avec un } \\
\text { technicien PA }\end{array}$ & $\mathbf{1 2 1 0 6 1 9 , 9 0 0 * *}$ & 585927,611 & 2,066 & 0,042 \\
$\begin{array}{l}\text { Accès au barrage } \\
\text { Age du Chef de }\end{array}$ & $\mathbf{9 5 7 4 5 6 , 4 7 8 *}$ & 568108,651 & 1,685 & 0,096 \\
ménage & $\mathbf{6 3 3 9 8 , 2 4 3}^{* *}$ & 30163,717 & 2,102 & 0,039
\end{tabular}




$\begin{array}{lcccc}\text { Etat climat } & \mathbf{1 1 3 1 8 7 8 , 0 0 3} * & 574836,735 & 1,969 & 0,052 \\ \text { Apparition épizootie } & -80341,954 & 665465,628 & -, 121 & 0,904 \\ \begin{array}{l}\text { Accès aux produits } \\ \text { vétérinaires }\end{array} & 410260,807 & 695028,151 & , 590 & 0,557\end{array}$

\begin{tabular}{lc}
$\begin{array}{l}\text { Récapitulatif } \\
\text { modèle }\end{array}$ & $\mathbf{R}^{2}=0,391$ \\
& $\mathbf{P}=0,009 * * *$ \\
& $N=85$ \\
\hline
\end{tabular}

*** significatif à $1 \%$; ** significatif à 5\% ; * significatif à 10\%

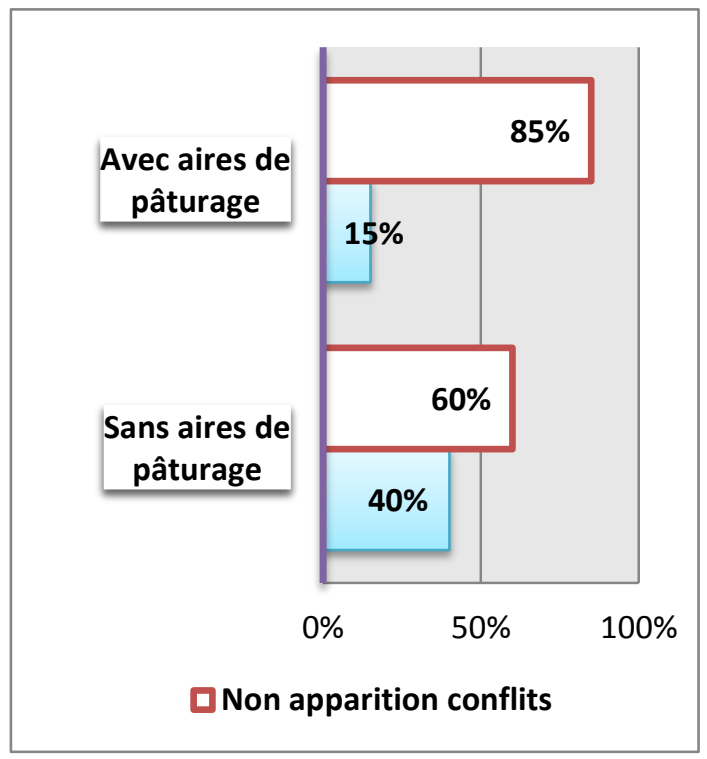

Figure 4 : Fréquence des conflits entre éleveurs et agriculteurs avec ou sans aires de pâturage.

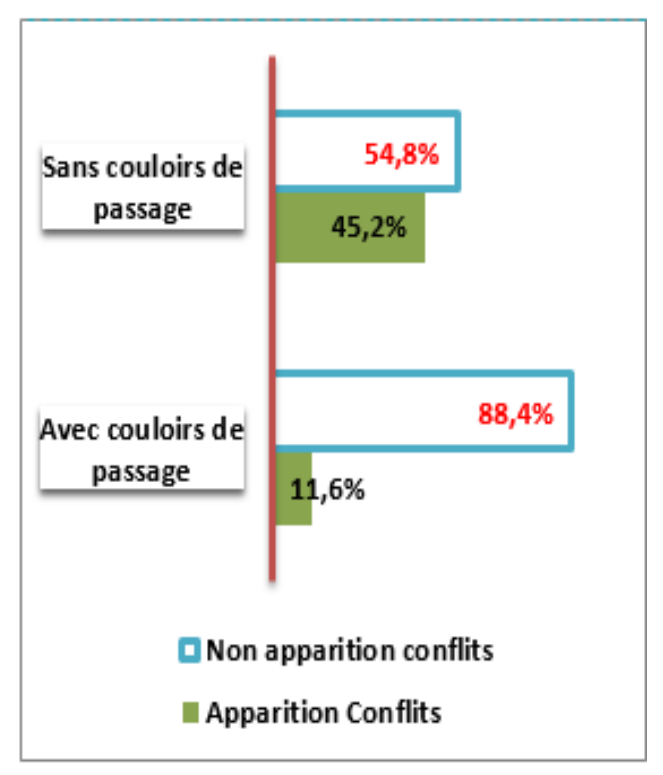

Figure 5: Fréquence des conflits entre éleveurs et agriculteurs avec ou sans couloirs de passage.

Tableau 5 : Résultats du modèle logistique binaire.

\begin{tabular}{|c|c|c|c|c|c|}
\hline Variables & $\mathbf{A}$ & Wald & $\operatorname{Exp}(\mathbf{B})$ & Effet marginal & $P$ de Signification \\
\hline Variable dépendante & & \multicolumn{4}{|c|}{ Apparition conflit ( $O=$ non ; $1=0 u i)$} \\
\hline Constante & 0,742 & 0,989 & 2,101 & 0,747 & 0,320 \\
\hline COMITRANS & $-1,239 *$ & 4,001 & 0,290 & 0,619 & 0,045 \\
\hline EMERORGPROEL & $-0,241$ & 0,164 & 0,786 & 0,594 & 0,685 \\
\hline LOISCOHA & 0,988 & 2,082 & 2,685 & 0,684 & 0,149 \\
\hline AIREPAT & $-1,568 *$ & 6,416 & 0,208 & 0,619 & 0,011 \\
\hline COULPASS & $-2,269 * *$ & 12,016 & 0,103 & 0,655 & 0,001 \\
\hline $\begin{array}{l}\text { Récapitulatif } \\
\text { modèle }\end{array}$ & & \multicolumn{4}{|c|}{$\begin{array}{c}\mathbf{R}^{2} \text { de Nagelkerke }=0,396 \\
\begin{array}{c}P=0,00001 * * \\
\text { N=85 }\end{array}\end{array}$} \\
\hline
\end{tabular}

** significatif à 1\%; * significatif à 5\% ; -2log-vraisemblance=73,766; R-deux de Cox \& Snell=0,276. 


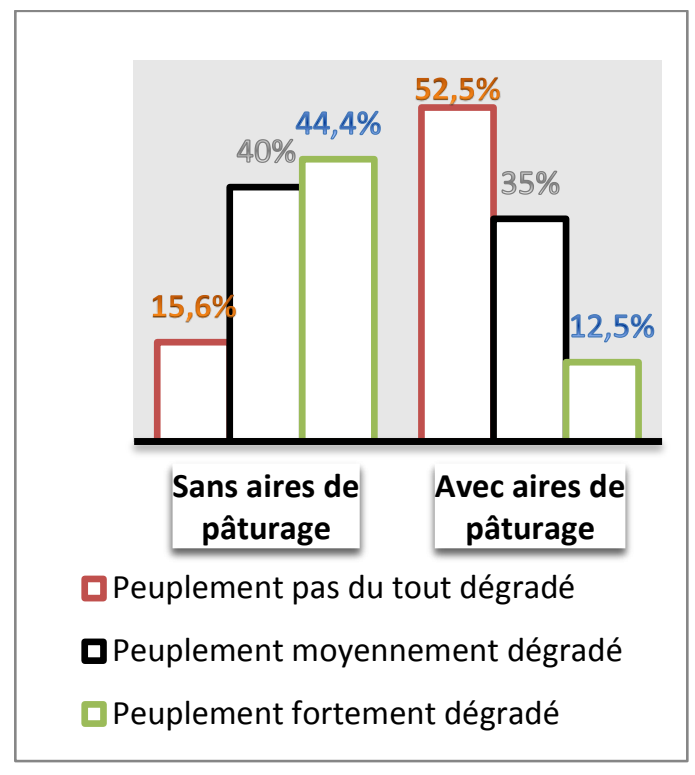

Figure 6: Perceptions sur l'état physique des peuplements forestiers à usage animal avec ou sans aires de pâturage.

\section{DISCUSSION}

Les résultats de cette étude montrent que l'accès aux barrages et retenues d'eau aménagées améliorent significativement tant les revenus des agriculteurs que ceux des éleveurs. En effet, les ressources hydrauliques sont d'une grande importance dans les systèmes de production végétale et animale en ce sens qu'elles permettent d'une part un bon développement des cultures, donc de bons rendements qui par ricochet induisent de revenus plus élevés. D'autre part, les ressources en eau garantissent un bon développement morphologique et un bon état de santé des animaux. Un troupeau qui accède sans difficultés à l'eau est prédisposé à se développer normalement si les autres conditions alimentaires sont réunies. Ce bon développement des animaux influencerait la taille (hauteur et poids) des animaux et la production du lait qui à leur tour feraient augmenter les revenus des éleveurs. Ces infrastructures ont donc des incidences positives sur les performances économiques des acteurs concernés. Ces résultats confirment les théories économiques qui établissent une corrélation positive entre l'output et les inputs. Toute chose étant égale

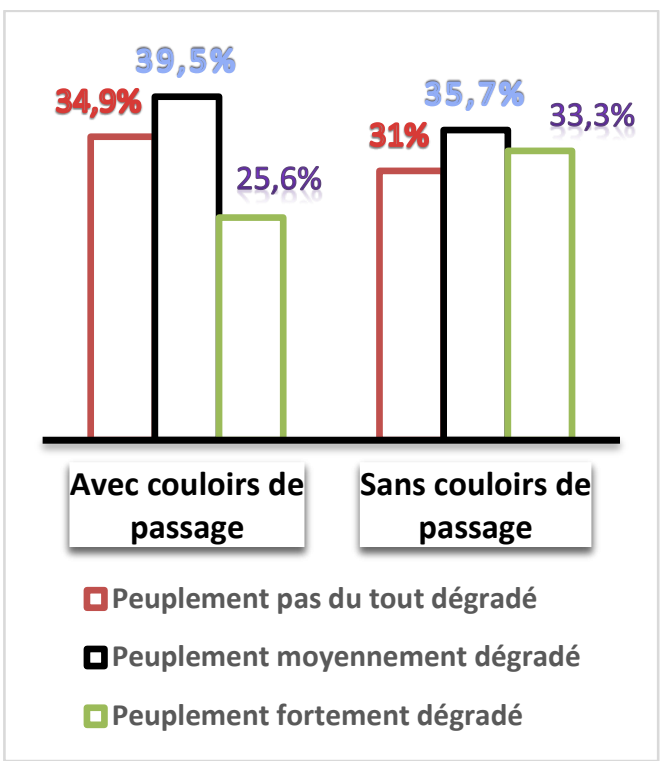

Figure 7 : Perceptions sur l'état physique des peuplements forestiers à usage animal avec ou sans aires de pâturage.

par ailleurs, l'accès aux inputs en quantité et en qualité suffisantes, améliorerait la production.

Cependant, l'amélioration des revenus des agriculteurs et éleveurs est aussi expliquée par d'autres facteurs tels que le contact avec un agent encadreur, l'accès à l'éducation et l'accès aux crédits agricoles pour ce qui est des agriculteurs; par l'âge de l'éleveur, le contact avec un technicien en production animale et l'état du climat pour le cas des éleveurs.

Etre en contact avec un agent encadreur est une source d'informations sur les itinéraires techniques améliorés de production, les intrants améliorés et certifiés. Un tel producteur dispose donc des connaissances actualisées sur les itinéraires techniques de production. Ainsi, ces performances productives (rendement, productivité, efficacité, etc.) se verraient boostées. D'où il ferait un profit plus important que ces homologues n'ayant pas accès à un agent d'encadrement. De même, un producteur instruit est plus ouvert aux innovations, adopterait plus facilement les technologies améliorées de production dans son exploitation que celui non instruit. Aussi, 
de tels producteurs appliqueraient-ils mieux dans leurs exploitations les meilleures techniques de production vulgarisées par la recherche. Une chose étant égale par ailleurs, leurs rendements seraient également plus élevés que ceux de leurs homologues non instruits qui négligeraient certaines technologies. La corrélation positive mise en évidence entre le crédit et le revenu des agriculteurs s'expliquerait par le fait que le crédit permettrait non seulement aux producteurs d'accéder aux intrants améliorés, mais aussi d'effectuer à bonne date les opérations culturales. Ce qui induirait une augmentation du rendement donc de leurs revenus.

L'influence positive de l'âge de l'éleveur sur son revenu pourrait s'expliquer par le fait qu'au fil des années, l'éleveur accumule de l'expérience en termes de de tenue de troupeaux. Il aurait capitalisé des connaissances endogènes d'élevage qui pourraient agir positivement sur la production de son cheptel et donc sur ses recettes. L'impact positif de l'état du climat sur le revenu des éleveurs serait dû au fait qu'une saison caractérisée par une bonne pluviométrie favoriserait la pousse des essences herbacée et forestière qui servent de fourrage aux animaux. Cette disponibilité en quantité suffisante de fourrage engendrerait une bonne performance productive des animaux et donc par ricochet une augmentation des revenus. L'accès à un technicien en production animale influence positivement le revenu des éleveurs du fait que les conseils en production animale et les informations en termes de produits vétérinaires efficaces auxquels ils accèdent auprès $\mathrm{du}$ technicien amélioreraient les performances productives de leurs cheptels et par ricochet leurs revenus.

Ces résultats corroborent ceux de Yabi et al. (2009) qui ont identifié l'accès à l'éducation formelle, le nombre d'années d'expérience, le contact avec un animateur de projet ou d'ONG, l'accès au crédit, l'utilisation d'une machine de concassage ou de mouture comme étant les variables déterminant l'efficacité économique des femmes transformatrices du beurre de karité au Nord-Bénin. La différence entre ces deux études réside dans les acteurs objet de l'étude et les indicateurs de mesure utilisés. Toutefois, l'un (efficacité) étant un déterminant de l'autre (revenu), ces facteurs peuvent également l'influencer.

Aussi, les résultats de cette étude ontils permis de conclure que les infrastructures pastorales et agropastorales ont des effets sociaux sur les communautés les abritant. Les couloirs de passage et les aires de pâturage atténuent les conflits entre éleveurs et agriculteurs dans les zones où ils sont installés. Etant des corridors officiellement définis qui canalisent les déplacements des troupeaux dans les zones agropastorales, en reliant les pâturages, les points d'eau et les zones de pacage, les couloirs de passage réduisent considérablement la probabilité d'apparition des conflits entre éleveurs et agriculteurs. La concurrence entre acteurs autour des ressources qui de plus en plus se raréfient, est gérée au mieux. Ces résultats viennent confirmer les conclusions de Ibrahim et al. (2018) et de Fielmua et al. (2014) qui stipulent que l'accès aux points d'eau et aux pâturages demeure le centre des conflits fonciers entre les différents groupes d'usagers dont la tendance est à l'appropriation par les groupes dominants. Ces infrastructures, de par leur installation et le respect des principes par les groupes d'acteurs, régulent l'usage des ressources par ceux-ci et par ricochet limitent les conflits. Cependant, contrairement aux auteurs cités plus haut et aux résultats de cette étude, Turner (2004) fait remarquer que les conflits entre agriculteurs et éleveurs ne s'interprètent pas seulement comme des conflits autour de ressources rares ou comme une défaillance de la gestion commune. Il déclare qu'il n'y a pas que des conflits d'intérêts entre les acteurs, mais que les relations entre groupes constituent également des dimensions morales et culturelles des conflits. Gangneron (2013) quant à lui aborde dans le même sens que Turner (2004) en montrant par exemple qu'au Mali, les conflits entre les agro-éleveurs et les Peuls transhumants dans la commune de Hombori 
sont liés à la maitrise territoriale entre les Songhaï disposant d'un pouvoir politique et les agro-éleveurs Bellahs des classes serviles.

L'étude a mis également en évidence le rôle crucial des comités de transhumance dans la fréquence des conflits. Il a été constaté que l'existence des comités de transhumance réduit la fréquence des conflits entre éleveurs et agriculteurs. Ce résultat serait dû aux effets de l'intermédiation que jouent de tels comités. En effet, dès qu'un cas de destruction de champs par les animaux est signalé par un quelconque éleveur ou agriculteur, le comité de transhumance entame les négociations avec la victime et procède au règlement à l'amiable des différends. Aussi, ce comité veille-t-il au respect des couloirs de passage et aires de pâturage de sorte que les agriculteurs ne s'y installent pas et que les éleveurs y fassent paitre leurs animaux. Cette veille permanente qu'assurent ces comités avec les organisations professionnelles d'éleveurs entraîne la baisse conséquente du taux d'apparition des conflits. Ce résultat s'apparente à ceux obtenu par Kiema et al., 2014 qui trouvent que le nonrespect des règles/lois dans les zones d'accueil et de transit, le manque de respect des normes sociales des zones d'accueil par les pasteurs sont aussi à l'origine de ces conflits. Certes, l'accès aux ressources communes constitue le principal motif des conflits entre éleveurs et agriculteurs, mais d'autres paramètres aussi bien physiques, moraux qu'institutionnels déterminent l'apparition de ces conflits.

Cette étude a également mis en évidence l'impact positif des infrastructures pastorales et agropastorales sur l'environnement, notamment sur certaines essences forestières à usage animal. Il a été constaté qu'avec les couloirs de passage et aires de pâturage, la pression sur le peuplement de certaines essences forestières diminue. Quarante-quatre pourcent (44\%) des enquêtés affirment que l'état du peuplement forestier à usage animal est fortement dégradé dans les zones sans aires de pâturage contre $12 \%$ qui le reconnaissent dans les zones où il y a les aires de pâturages. Il en est de même pour les couloirs de passage où $33 \%$ constatent une forte dégradation des essences forestières dans les zones sans couloirs contre $25 \%$ dans celles à couloirs de passage. Les essences les plus attaquées sont Afzelia africana, Khaya senegalensis et Pterocarpus erinaceus. Lesse et al. (2015), au terme de leur étude sur la transhumance au Bénin ont conclu que les essences forestières telles que Afzelia africana, Khaya senegalensis et Pterocarpus erinaceus sont les arbres fourragers les plus utilisés pendant la saison sèche par les éleveurs à travers le pâturage aérien qu'ils pratiquent. Ce faisant, elles (essences forestières) sont plus exposées aux attaques des éleveurs qu'ils soient transhumants ou sédentaires. D'où leur forte dégradation dans les espaces où ces infrastructures pastorales et agropastorales n'existent pas. Les couloirs de passage et aires de pâturage, de par la délimitation des corridors de circulation et zones où doivent paître les animaux, réduisent donc l'exposition de ces essences aux pressions pastorales. Toutefois, il faut signaler que l'état de dégradation des peuplements des essences forestières est aussi expliqué par les actions anthropiques (utilisations pour des fins médicinales, utilisation domestique : bois de chauffe et d'œuvre; les feux de brousse incontrôlés; etc.) et les changements climatiques. Umutoni et Ayantunde (2018) sont aussi parvenus à de telles conclusions au terme de leur étude qui s'est focalisée sur la perception des acteurs ruraux sur les effets de la transhumance sur la gestion des ressources naturelles au Sud du Mali. Soixante-quinze pourcent $(75 \%)$ des enquêtés sur les deux sites d'étude établissent un lien entre la rareté des ressources en eau, la dégradation des essences végétales et l'accroissement de l'effectif des troupeaux transhumants. Cinquante pourcent (50\%) des producteurs et des communautés tirant leurs moyens de survie des ressources naturelles lient la dégradation de la végétation aux effets de la transhumance. Les éleveurs eux affirment que cette dégradation est due aux changements climatiques.

\section{Conclusion}

Le présent papier dont l'objectif est d'évaluer les effets socio-économiques et 
environnementaux des infrastructures pastorales et agropastorales sur les communautés les abritant a permis de conclure que les ouvrages pastoraux et agropastoraux ont effectivement des incidences socio-économiques et environnementales sur les populations des zones où ils sont installés. Non seulement elles contribuent à améliorer les revenus des utilisateurs directs (éleveurs et agriculteurs), elles participent également dans une certaine mesure à l'apaisement du climat social au sein des communautés et préservent certaines essences forestières. Ces infrastructures pastorales et agropastorales n'expliquent pas à elles seules ces incidences positives. Plusieurs autres facteurs contribuent à cette amélioration de revenus, à l'apaisement du climat social au sein des communautés et à la préservation de l'environnement. Le contact avec un agent encadreur, l'accès à l'éducation, l'accès aux crédits agricoles contribuent au même titre que les infrastructures hydrauliques à l'amélioration du revenu des producteurs. L'âge de l'éleveur, le contact avec un technicien en production animale et l'état du climat déterminent également le revenu des éleveurs. Les fréquences d'apparition des conflits entre les éleveurs et les agriculteurs se voient diminuer avec le traçage des couloirs de passage, la délimitation des aires de pâturage et la mise en place des comités de transhumance. Les actions anthropiques (prélèvement pour des fins médicinales, domestiques, les feux de brousse incontrôlés, etc.) et les changements climatiques influencent également le degré de dégradation des essences forestières. Cependant, il a été noté le non-respect des couloirs et des aires de pâturage aussi bien par les éleveurs que par les agriculteurs qui se traduit d'une part par le débordement des animaux des couloirs et le pâturage dans les champs et d'autre part par l'installation des agriculteurs sur ces couloirs et aires réservés aux activités pastorales. Ce qui justifie l'apparition de quelques conflits dans les zones où ces infrastructures sont réalisées. Aussi, a-t-il été constaté que les infrastructures hydrauliques non seulement sont en nombre insuffisant, mais mal entretenues du fait du défaut de gestion. Alors pour plus d'impact sur les utilisateurs, il faudra mieux gérer ces infrastructures et mettre en place les instances réglementant la cohabitation des éleveurs et agriculteurs et veiller au respect des règles. Il faudra également revoir les modes de gestion des infrastructures hydrauliques et marchandes pour garantir leur durabilité pour le bonheur des utilisateurs.

\section{CONFLIT D'INTERETS}

Les auteurs de ce papier ne déclarent aucun conflit d'intérêt. Tous ont contribué à son élaboration.

\section{CONTRIBUTIONS DES AUTEURS}

AIM et AM ont contribué à la collecte, à l'analyse des données et à la rédaction du papier. YJ, DH, HM ont apporté leurs observations sur le contenu du papier; BG a supervisé et corrigé le papier de la collecte à la publication.

\section{REMERCIEMENTS}

Les auteurs remercient les personnes enquêtées pendant la phase de la collecte des données pour leurs oreilles attentives qu'elles ont prêtées aux questions à elles posées.

\section{REFERENCES}

Abdoulaye IM, Ayena M, Yabi AJ, Dedehouanou H, Biaou G, Houinato M. 2018. Gouvernance locale des infrastructures pastorales et agropastorales dans le Borgou au NordEst du Benin: Quels modes pour une gestion durable des infrastructures? Int. J. Innov. Appl. Stud., 24 (3): 1312-1323. DOI: $\quad$ http://www.ijias.issrjournals.org/abstract.php?article=IJIAS18-126-14

Adekambi SA. 2005. Impact de l'adoption des variétés améliorées de riz sur la scolarisation et la santé des enfants au Benin: cas du Département des Collines. (Rapport d'étude). Porto-Novo; p 100

Archambaud L, Tidjani I. 2016. Etude des réalités agro-pastorales en Ouham 
(Rapport d'étude pour le renforcement des capacités de résilience des populations en Ouham). DOI: https://fscluster.org/sites/default/files/do cuments/2016_09_etude_agropastorale_ ouham_acf_0.pdf.

Ayantunde AA, Asse R, Said MY, Fall A. 2014. Transhumant pastoralism, sustainable management of natural resources and endemic ruminant livestock in the sub-humid zone of West Africa. Env. Dev Sustain., 16(5): 10971117.

DOI: https://dx.doi.org/10.1007/s10668014-9515-z

Brunet R, Ferras R, Théry H. 1993. Les mots de la géographie. Dictionnaire critique, RECLUS. France.

Cocharne W, Rubin P. 1973. Controlling bias in observational studies. Sankyha, the Indian Journal of Statitics, Series A., 35(4): 417-446. DOI: https://www.jstor.org/stable/25049893

Dangbo FA, Adjonou K, Kokou K, Blaser J. 2019. The socio-economic contribution of Detarium senegalense seeds to rural livelihoods in Togo (West Africa). Int. J. Biol. Chem. Sci., 13(3): 1582-1595. https://doi.org/10.4314/ijbcs.v13i3.30

Delville PL. 2006. Conditions pour une gestion décentralisée des ressources naturelles. Entre "community failures", "market failures" et "state failures", construire de nouveaux 'communs'. Alain Bertrand; Alain Karsenty; Pierre Montagne. L'Etat et la gestion locale durable des forêts en Afrique francophone et à Madagascar. 200621.

Diagne A. 2003. Evaluation de l'impact: synthèse des développements méthodologiques récents. Presented at the ADRAO, Conakry, p. 15.

Djenontin JA. 2010. Dynamique des stratégies et des pratiques d'utilisation des parcours naturels pour l'alimentation des troupeaux bovins au Nord-Est du Bénin (Rapport d'étude). Benin.

Fall AA. 2006. Impact du crédit sur le revenu des riziculteurs de la vallée du fleuve Sénégal. Thèse de Doctorat. Ecole
Nationale Supérieure de Montpellier, Université Montpellier I, France. Montpelier, France, 350 p.

Fielmua N, Bandie DBR, Ziemah KM. 2014. Managing Pastoralism and Water Rights in the Upper West Region of Ghana: A Blame Game Among Actors. J. Sustain. Dev., 7(1): 72-84. DOI: 10.5539/jsd.v7n1p72

Gangneron F. 2013. Ressources pastorales et territorialité chez les agro-éleveurs sahéliens du Gourma des buttes. Vertigo Rev. Électronique En Sci. Environ., 13 (3): DOI: https://journals.openedition.org/vertigo/1 4427

Heckman J. 1990. Varieties of Selection Bias. Am Eco Rev, 80(2): 313-318. DOI: http://www.sciencedirect.com/science/art icle/pii/S0304-4076(04)00073-9

Ibrahim H, Mormont M, Stassart MP, Yamba B. 2018. Pastoralisme et politiques développementalistes : états de lieux sur les systèmes de tenure foncière pastorale en Afrique Subsaharienne. Vertigo Rev. Électronique En Sci. Environ., 15.

Ibrahim, Habibou, 2016. Etude de l'émergence des organisations pastorales et de leur rôle dans les processus de gestion partagée de la vallée de la Tarka dans les départements de Bermo et Dakoro (Centre sud du Niger) Recherche Google [WWW Document].

IFAD 1998. Managing for impact in rural development: a guide for project $\mathrm{M} \& \mathrm{E}$.

Kiema A, Bambara G, Tontibomma Zampaligré N. 2014. Transhumance et gestion des ressources naturelles au Sahel: contraintes et perspectives face aux mutations des systèmes de productions pastorales. Vertigo Rev. Électronique En Sci. Environ., 14(3). DOI:

https://journals.openedition.org/vertigo/1 5404.

Lesse P, Houinato RBM, Djenontin AJ, Dossa H, Yabi B, Toko I, Tente B, Sinsin B. 2015. Transhumance en République du Bénin : états des lieux et contraintes. Int 
J Biol Chem Sci, 9(5): 2668-2681. DOI: http://dx.doi.org/10.4314/ijbcs.v9i5.37

MAEP. 2010. Annuaire statistique du Ministère de l'Agriculture de l'Elevage et de la Pêche.

Oyono PR, Ribot JC, Larson AM. 2007. Or Vert et Or Noir dans le Cameroun Rural: Ressources Naturelles pour la Gouvernance Locale, la Justice et la Durabilité. Washington D.C., WRI, 96 p. Paraïso A, Yabi AJ, Sossou A, Zoumarou WN, Yègbémey NR. 2012. Rentabilité économique et financière de la production cotonnière à Ouaké au NordOuest du Benin. Ann. Sci. Agron., 16: 114.

Savi AD, Adekambi SA, Adegbola PY, Glito M. 2008. Impacts socio-économiques des technologies améliorées d'alimentation des petits ruminants au Benin (Rapport d'étude). PAPA/PortoNovo ; Benin, $90 \mathrm{p}$.

Sgahaier M. 2010. Etude de la gouvernance des ressources naturelles dans les oasis: Cas des oasis en Tunisie (étude), UICN, $69 \mathrm{p}$.

Sounon KLSA, Aho E, Lesse P, Ickowicz A, Messad S, Lesnoff M, Houinato MRB. 2019. Evaluation de la valeur économique directe de l'élevage bovin au nord-Bénin. Int. J. Biol. Chem. Sci., 13(1): $8-17 . \quad$ DOI: https://doi.org/10.4314/ijbcs.v13i1.2

Tama C, Mossi A. 2011. Rapport de l'état des lieux des infrastructures et aménagements agropastoraux dans quelques localités de la zone d'intervention de ASPAP (Le département du Borgou et quelques communes de l'Alibori) $76 \mathrm{p}$.

Turner DM. 2004. Political ecology and the moral dimensions of "resources conflicts" the case of farmer-herder conflicts in the Sahel. Political Geography, 23(7): 863-889. DOI : 10.1016/j.polgeo.2004.05.009.

Umutoni C, Ayantunde AA. 2018. Perceived effects of transhumant practices on natural resource management in southern Mali. Pastoralism, 8(8). DOI: https://doi.org/10.1186/s13570-0180115-7

Umutoni C, Ayantunde AA, Sawadogo GJ. 2016. Connaissance locale des pratiques de la transhumance dans la zone soudano-sahélienne du Mali. Rev Elev Med Vet Pays Trop, 69 (2): 53-61.

Yabi AJ, Adegbola PY, Tovignan DS, Ahouandjinou MC, Adekambi SA. 2014. Impact of the adoption of semi mechanized technologies of shea processing on rural women's income in northern Benin (West africa). J. Dev. Agric. Econ., 66: 249-256. DOI: 10.5897/JDAE 12.123

Yabi AJ, Ouinsavi C, Sokpon N. 2009. Facteurs d'efficacité technicoéconomique de transformation du karité en beurre au Nord-Bénin. Ann Univ Lomé Sér. Sc Eco Gest, 3: 23-44.

Yabi AJ, Paraïso A, Yègbémey NR, Chanou P. 2012. Rentabilité économique des systèmes rizicoles de la commune de Malanville au Nord-est du Benin. Bulletin de la Recherche Agronomique $d u$ Bénin, Numéro Spéc. Prod. Végétales Anim. Econ. Sociol. Rural. 12. Youssao AKI, Dahouda M, Attakpa EY, Koutinhouin GB, Ahounou GS, Toleba SS, Balogoun BS. 2013. Diversité des systèmes d'élevages de bovins de race bovine Borgou dans la zone soudanienne du Bénin. Int J Biol Chem Sci, 7(1): 125146.

DOI: http://dx.doi.org/10.4314/ijbcs.v7i1.11. 